\title{
BioGlue cerebral embolism following acute type A aortic dissection repair
}

\author{
Manabu Yamasaki ${ }^{1}$, Kohei $\mathrm{Abe}^{2}$, Ryota Nakamura ${ }^{2}$, Rihito Tamaki ${ }^{2}$, and Hiroyasu \\ Misumi $^{2}$ \\ ${ }^{1}$ St Lukes's international hospital \\ ${ }^{2}$ St Luke's International University
}

June 29, 2021

\begin{abstract}
The outcomes of surgery for Acute Stanford type A aortic dissection (ATAAD) have significantly improved in recent decades due to advances in surgical techniques and, surgical adhesives such as BioGlue. However, this convenient material can sometimes cause complications such as thrombotic embolism and pseudoaneurysm. Here, we present the case of a 61-year-old male with ATAAD who successfully underwent total arch replacement. Five days after the surgery, he collapsed due to right hemiplegia. We immediately performed cerebral thrombectomy and removed the thrombotic embolus caused by BioGlue, which was used to obliterate the false lumen of the dissected aorta during ATAAD repair.
\end{abstract}

\section{Introduction}

On account of a variety of hemostatic agents, adhesives, and sealants, the outcomes of surgery for Acute Stanford type A aortic dissection (ATAAD) repair have improved greatly. BioGlue (Cryolife Kennesaw, Georgia, USA) is an adhesive (independent of the coagulation cascade) which has hemostatic properties and also strengthens and holds tissues together at sites of complex anastomoses ${ }^{1}$. There are some reports about complications associated with the use of BioGlue, such as acute cerebral embolism ${ }^{1}$, myocardial infarction ${ }^{2}$, and pseudoaneurysm ${ }^{3}$. Here, we report a rare case of a patient with embolism associated with BioGlue after ATAAD repair, treated by cerebral thrombectomy.

\section{Case Report}

A previously healthy 61-year-old male was referred to our hospital for sudden chest pain. Chest computed tomography (CT) revealed ATAAD. We performed emergency total arch replacement for ATAAD using the elephant trunk technique under cardiopulmonary bypass and ante-grade selective cerebral perfusion (Figure $1 \mathrm{a}$ and $\mathrm{b}$ ). The patient was extubated on postoperative day 1 and started the rehabilitation. However, he collapsed with right hemiplegia on postoperative day 5 . We performed three-dimensional cerebral CT that revealed occlusion of the left anterior cerebral artery (Figure 2). Cerebral thrombectomy was performed by cerebral interventionalists. Pathology reports revealed a yellowish material that was collected with the fresh thrombus to be consistent with BioGlue (Figure $3 \mathrm{a}$ and $\mathrm{b}$ ).

\section{Comment}

Advances in surgical techniques and the development of medical organizations has enabled improvements in the outcome of surgeries for ATAAD ${ }^{4}$. However, it remains a life-threatening medical emergency associated with high morbidity and mortality. It is essential to explore ways to reduce perioperative cerebral events after ATAAD repair as they are associated with poor early outcomes ${ }^{5}$. A few possible mechanisms of perioperative 
cerebral events after ATAAD repair include partial or complete occlusion of the arch vessels by the intimalmedial flap (cerebral malperfusion), hypoxic encephalopathy secondary to shock or tamponade, and/or brain embolism from thrombus in the false lumen ${ }^{6.7}$. Currently, on observing partial thrombosis in the false lumen of the ascending aorta of a patient with ATAAD, we perform direct cannulation of the malperfused carotid artery and select the subclavian artery as cannulation site, to avoid perioperative cerebral events after ATAAD repair. In our case, we performed true lumen cannulation of the ascending aorta using the Seldinger technique as we did not observe any thromboses in the false lumen. Although our patient woke without any neurological dysfunction on postoperative day 1 , he collapsed with right hemiplegia due to thrombotic embolism associated with BioGlue a few days after the surgery.

There have been some reports on the complications associated with the use of BioGlue, such as stroke, coronary embolism, and pseudoaneurysm formation ${ }^{1-3}$. Carrel et al. reported three possible mechanisms by which surgical adhesives such as BioGlue can cause thrombotic embolism ${ }^{8}:$ (1) direct spillage of glue into the true lumen (despite precautions); (2) escape of glue through distal reentry sites into the true lumen; and (3) secondary mobilization of glue particles through suture-line needle holes. With the third mechanism, we cannot avoid embolic events even with careful application of BioGlue. Considering that the embolic event in our case occurred a few days after surgery, we believe that the third mechanism may have played a role, though we cannot prove it. Cerebral thrombectomy is not usually indicated for embolic events during surgery. Therefore, we believe our case indicates that cerebral embolic stroke due to use of BioGlue may occur more frequently in clinical practice.

\section{References}

LeMaire SA, Carter SA, Won T, Wang X, Conklin LD, Coselli JS. The threat of adhesive embolization: BioGlue leaks through needle holes in aortic tissue and prosthetic grafts. Ann Thorac Surg . 2005;80(1):106111.

Mahmood Z, Cook DS, Luckraz H, O'Keefe P. Fatal right ventricular infarction caused by

Bioglue coronary embolism. J Thorac Cardiovasc Surg. 2004;128(5): 770-771.

1. Luk A, David TE, Butany J. Complications of Bioglue postsurgery for aortic dissections and aortic valve replacement. J Clin Pathol . 2012; 65(11):1008-1012.

2. Abe T, Yamamoto H, Miyata H, et al. Patient trends and outcomes of surgery for type A acute aortic dissection in Japan: an analysis of more than 10000 patients from the Japan Cardiovascular Surgery Database. Eur J Cardiothorac Surg . 2020; 57(4):660-667.

3. Conzelmann LO, Hoffmann I, Blettner M, et al. Analysis of risk factors for neurological dysfunction in patients with acute aortic dissection type A: data from the German Registry for Acute Aortic Dissection type A (GERAADA). Eur J Cardiothorac Surg . 2012; 42(3):557-565.

6. Czerny M, Schoenhoff F, Etz C, et al. The Impact of Pre-Operative Malperfusion on Outcome in Acute Type A Aortic Dissection: Results From the GERAADA Registry. J Am Coll Cardiol . 2015; 65(24): 2628-2635.

7. Geirsson A, Szeto WY, Pochettino A, et al. Significance of malperfusion syndromes prior to contemporary surgical repair for acute type A dissection: outcomes and need for additional revascularizations.Eur $J$ Cardiothorac Surg . 2007; 32(2): 255-262.

8. Carrel T, Maurer M, Tkebuchava T, Niederhäuser U, Schneider J, Turina MI. Embolization of biologic glue during repair of aortic dissection. Ann Thorac Surg. 1995;60(4):1118-1120.

\section{Patient consent statement}

The present patient provided informed consent.

\section{Conflict of Interest}

The authors declare that there is no conflict of interest. 
Figure legends

Fig. 1: Chest computed tomography (CT) shows (a): acute Stanford type A aortic dissection (ATAAD). (b): three-dimensional CT of ATAAD.

Fig. 2: Brain three-dimensional CT shows occlusion of the left anterior cerebral artery (black arrow).

Fig. 3: (a, b) BioGlue collected by cerebral thrombectomy (yellow arrow). (c) Pathological image revealed a structureless material with scarce cellular components that stained uniformly pink with hematoxylin and eosin stain

\section{Hosted file}

Fig 1 Bioglue.pdf available at https://authorea.com/users/387737/articles/528357-biogluecerebral-embolism-following-acute-type-a-aortic-dissection-repair

\section{Hosted file}

Fig 2 Bioglue JCS.pdf available at https://authorea.com/users/387737/articles/528357-biogluecerebral-embolism-following-acute-type-a-aortic-dissection-repair

\section{Hosted file}

Fig 3 Bioglue JCS PDF.pdf available at https://authorea.com/users/387737/articles/528357bioglue-cerebral-embolism-following-acute-type-a-aortic-dissection-repair 